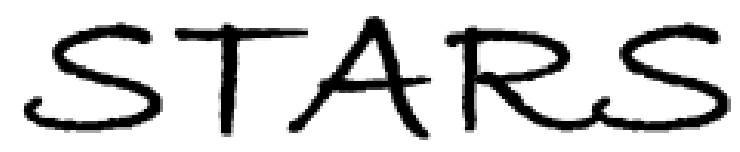

University of Central Florida

STARS

$1-1-2013$

\title{
On A Calderon Problem In Frequency Differential Electrical Impedance Tomography
}

Sungwhan Kim

Alexandru Tamasan

University of Central Florida

Find similar works at: https://stars.library.ucf.edu/facultybib2010

University of Central Florida Libraries http://library.ucf.edu

This Article is brought to you for free and open access by the Faculty Bibliography at STARS. It has been accepted for inclusion in Faculty Bibliography 2010 s by an authorized administrator of STARS. For more information, please contact STARS@ucf.edu.

\section{Recommended Citation}

Kim, Sungwhan and Tamasan, Alexandru, "On A Calderon Problem In Frequency Differential Electrical Impedance Tomography" (2013). Faculty Bibliography 2010s. 4210.

https://stars.library.ucf.edu/facultybib2010/4210

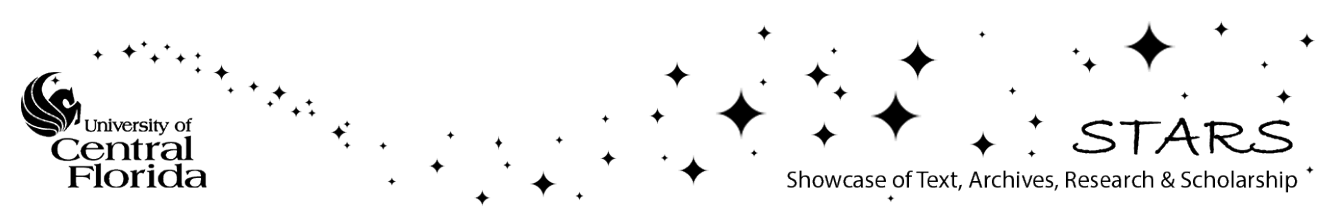




\title{
ON A CALDERÓN PROBLEM IN FREQUENCY DIFFERENTIAL ELECTRICAL IMPEDANCE TOMOGRAPHY*
}

\author{
SUNGWHAN $\mathrm{KIM}^{\dagger}$ AND ALEXANDRU TAMASAN ${ }^{\ddagger}$
}

\begin{abstract}
Recent research in electrical impedance tomography produced images of biological tissue from frequency differential boundary voltages and corresponding currents. Physically one is to recover the electrical conductivity $\sigma$ and permittivity $\epsilon$ from the frequency differential boundary data. Let $\gamma=\sigma+i \omega \epsilon$ denote the complex admittivity, $\Lambda_{\gamma}$ be the corresponding Dirichlet-to-Neumann map, and $\left.\frac{d \Lambda_{\gamma}}{d \omega}\right|_{\omega=0}$ be its frequency differential at $\omega=0$. If $\sigma \in C^{1,1}(\bar{\Omega})$ is constant near the boundary and $\epsilon \in C_{0}^{1,1}(\Omega)$, we show that $\left.\frac{d \Lambda_{\gamma}}{d \omega}\right|_{\omega=0}$ uniquely determines $\nabla \cdot(\nabla \epsilon-\epsilon \nabla \ln \sigma) / \sigma$ inside $\Omega$. In addition, if $\left.\Lambda_{\gamma}\right|_{\omega=0}$ is also known, then $\epsilon$ and $\sigma$ can be reconstructed inside. The method of proof uses the complex geometrical optics solutions.
\end{abstract}

Key words. Calderón problem, frequency differential electrical impedance tomography, complex geometrical optics

AMS subject classifications. 35R30, 35J25, 65N21

DOI. $10.1137 / 130904739$

1. Introduction. Electrical impedance tomography (EIT) aims to determine the electrical conductivity $\sigma$ and permittivity distribution $\epsilon$ of a body from surface electrical measurements of voltages and corresponding currents. One major application is in medical imaging, where the change of the electrical properties of biological tissues with their physiological and pathological conditions is used to provide diagnostic information. Driven by its applications, considerable progress in both the engineering and mathematical facets of EIT has been achieved, and its development can be traced over the past two decades in the reviews [6, 3, 8, 2, 19].

We consider a conducting bounded body $\Omega \subset \mathbb{R}^{n}, n \geq 3$, with $C^{1,1}$-boundary $\partial \Omega$. Its conductivity distribution $\sigma \in C^{1,1}(\bar{\Omega})$ is bounded away from zero, and its permittivity distribution $\epsilon \in C^{1,1}(\bar{\Omega})$. We assume that $\sigma$ is constant near the boundary and $\epsilon$ is supported in $\Omega$ (these assumptions may be relaxed as explained later).

For a real valued function $f \in H^{1 / 2}(\partial \Omega)$ and an angular frequency $\omega$ the sinusoidal voltage $f(x) \cos (\omega t)$ is imposed at the boundary $\partial \Omega$. Then a time harmonic complex voltage potential $u_{\omega}$ distributes inside according to the problem

$$
\nabla \cdot(\sigma+i \epsilon \omega) \nabla u_{\omega}=0 \text { in } \Omega \text { and }\left.u_{\omega}\right|_{\partial \Omega}=f .
$$

The problem (1.1) has a unique complex (voltage potential) solution $u_{\omega} \in H^{1}(\Omega)$; see also Theorem 3.1 below. The exiting current

$$
g_{\omega}:=(\sigma+i \omega \epsilon) \mathbf{n} \cdot \nabla u_{\omega}
$$

is measured ( $\mathbf{n}$ is the unit outward normal) at the boundary, to define the Dirichlet-

*Received by the editors January 4, 2013; accepted for publication (in revised form) July 8, 2013; published electronically September 12, 2013.

http://www.siam.org/journals/sima/45-5/90473.html

${ }^{\dagger}$ Division of Liberal Arts, Hanbat National University, Daejeon 305-719, Korea (sungwhan@ hanbat.ac.kr). This author was supported by Hanbat National University and by the 2007 Faculty Fund of Hanbat National University.

$\ddagger$ Department of Mathematics, University of Central Florida, Orlando, FL 32816 (tamasan@ math.ucf.edu). This author was supported in part by NSF grant DMS-1312883.

2700 
to-Neumann map

$$
\Lambda_{\sigma+i \epsilon \omega}: f \mapsto g_{\omega} \in H^{-1 / 2}(\partial \Omega) .
$$

Originally formulated by Calderón [5] at the $\omega=0$ frequency, the goal in EIT is to determine $\sigma$ and $\epsilon$ from knowledge of $\Lambda_{\sigma+i \epsilon \omega}$. At zero frequency only $\sigma$ is sought; in such a case the corresponding voltage potential $v_{0}$ is real valued and solves

$$
\nabla \cdot\left(\sigma \nabla v_{0}\right)=0 \text { in } \Omega \text { and }\left.v_{0}\right|_{\partial \Omega}=f .
$$

The Calderón problem (at $\omega=0$ ) has been mostly settled in the affirmative and we refer to [19] for a state of the art solution. Of relevance to our work here, we mention the breakthrough result in [18], where $\Lambda_{\sigma}:=\left.\Lambda_{\gamma}\right|_{\omega=0}$ is shown to uniquely determine $\sigma$ in three or higher dimensions, and the reconstruction method in [14] which allows for the $C^{1,1}$-regularity assumed here. We note however that, while not explicitly stated, the results in $[18,14]$ extend to the complex admittivity $\gamma=\sigma+i \omega \epsilon$ to show that $\Lambda_{\gamma}$ uniquely determines $\gamma$ in three dimensions or higher. The analogous results in two dimensions at $\omega=0$ were obtained in [15], with a nontrivial refinement in [1]. Also in two dimensions, but at an arbitrary fixed frequency $\omega$ (not necessarily zero) $\Lambda_{\gamma}$ was shown recently to uniquely determine the complex admittivity $\gamma$ in [4] (a previous result in [7] recovered $\gamma$ for a sufficiently small imaginary part $\omega \epsilon)$.

Recent research in $[9,11,12,16,17]$ produce physiologically relevant images by using the frequency dependent behavior of the complex potential $u_{\omega}$. These new methods are known as frequency differential electrical impedance tomography (fdEIT). Physically one imposes boundary voltages at two distinct frequencies and measures a difference between corresponding boundary exit currents. Despite the apparent usefulness in medical diagnostics, the quantities behind the images in fdEIT are not so well understood. In this paper we take a first step towards explaining what can be quantitatively obtained by fdEIT. We formulate the problem in terms of the frequency differential operator at the boundary: What can be obtained from knowledge of $\left.\frac{d \Lambda_{\gamma}}{d \omega}\right|_{\omega=0}$ ?

To simplify notation, let $\mathcal{D}$ be defined for real valued functions $f \in H^{1 / 2}(\partial \Omega)$ by

$$
\mathcal{D}(f):=\left.\frac{d}{d \omega}\right|_{\omega=0} \Lambda_{\sigma+i \omega \epsilon}(f),
$$

and then extended by complex linearity:

$$
\mathcal{D}(f+i g):=\mathcal{D}(f)+i \mathcal{D}(g) .
$$

Our main result, Theorem 2.2, shows that $\mathcal{D}: H^{1 / 2}(\Omega) \rightarrow H^{-1 / 2}(\Omega)$ is a well-defined bounded operator which uniquely determines the function $\nabla \cdot(\nabla \epsilon-\epsilon \nabla \ln \sigma) / \sigma$ inside, in three or higher dimensions. The method of proof uses the complex geometrical optics solutions in [18]. We note that only the action of $\mathcal{D}$ on real valued functions is needed.

If, in addition, the Dirichlet-to-Neumann map $\Lambda_{\sigma}:=\left.\Lambda_{\gamma}\right|_{\omega=0}$ is also known, we are able to recover separately the conductivity $\sigma$ and the permittivity $\epsilon$ inside $\Omega$. Moreover, in this case we do not need to assume $\sigma$ constant near the boundary. Specifically, if $\sigma \in C^{1,1}(\bar{\Omega})$ then its boundary values $\left.\sigma\right|_{\partial \Omega}$ and its normal derivative $\left.\frac{\partial \sigma}{\partial \mathbf{n}}\right|_{\partial \Omega}$ can be recovered from $\Lambda_{\sigma}$ as shown in [14] for this regularity (and earlier in [13] and [18] for $C^{\infty}$-conductivity). Then $\sigma$ can be extended with preserved $C^{1,1}$-regularity 
to the whole space while making it constant near the boundary $\partial \tilde{\Omega}$ of a neighborhood $\tilde{\Omega}$ of $\Omega$. As shown in [15] the Dirichlet-to-Neumann map can be transferred from $\partial \Omega$ to $\partial \tilde{\Omega}$ (the fact that we deal with a complex valued coefficient does not change the proof from the real case). Therefore the assumption that $\sigma$ is constant near the boundary $\partial \Omega$ does not restrict generality. Similarly, the assumption that $\epsilon$ has compact support in $\Omega$ can be replaced by the knowledge of the boundary values $\left.\epsilon\right|_{\partial \Omega}$ and its normal derivative $\left.\frac{\partial \epsilon}{\partial \mathbf{n}}\right|_{\partial \Omega}$; see also (4.4).

With $v_{\omega}:=\Re\left(u_{\omega}\right)$ and $h_{\omega}:=\Im\left(u_{\omega}\right)$ denoting the real, respectively, imaginary part of the voltage potential obtained for a real valued boundary data $f$, the problem (1.1) can be rewritten as a Dirichlet problem for the coupled system:

$$
\nabla \cdot\left[\left(\begin{array}{cc}
\sigma & -\omega \epsilon \\
\omega \epsilon & \sigma
\end{array}\right)\left(\begin{array}{c}
\nabla v_{\omega} \\
\nabla h_{\omega}
\end{array}\right)\right]=\left(\begin{array}{l}
0 \\
0
\end{array}\right) \text { in } \Omega,\left.\left(\begin{array}{l}
v_{\omega} \\
h_{\omega}
\end{array}\right)\right|_{\partial \Omega}=\left(\begin{array}{l}
f \\
0
\end{array}\right) .
$$

Not only do $v_{\omega}$ and $h_{\omega}$ have a nonlinear dependence on the conductivity $\sigma$, permittivity $\epsilon$, and angular frequency $\omega$, but also their intrinsic mutual relation makes this dependence difficult to investigate directly from (1.5).

Key to this work, in Theorem 3.1 we identify the regime of frequencies

$$
|\omega|<\left\|\frac{\epsilon}{\sigma}\right\|_{L^{\infty}(\Omega)}^{-1}
$$

in which the family of operators $\omega \mapsto \Lambda_{\sigma+i \epsilon \omega}$ is analytic in the strong operator topology (from $H^{1 / 2}(\Omega)$ to $H^{-1 / 2}(\Omega)$ ). This analytic dependence allows for a recurrence type of decoupling. Also the frequency differential boundary operator can be made more explicit:

$$
\mathcal{D}(f)=i \epsilon \frac{\partial v_{0}}{\partial \mathbf{n}}+\left.i \sigma \frac{d}{d \omega}\right|_{\omega=0} \frac{\partial h_{\omega}}{\partial \mathbf{n}}=\left.i \frac{\epsilon}{\sigma}\right|_{\partial \Omega} \Lambda_{\sigma}(f)+\left.i \sigma \frac{d}{d \omega}\right|_{\omega=0} \frac{\partial h_{\omega}}{\partial \mathbf{n}} .
$$

2. Statement of results. The main result is formulated in terms of the complex geometrical optics solutions of Sylvester and Uhlmann in [18] whose existence is recalled below both for convenience and to set notation. The coefficients $\sigma$, and $\epsilon$ assumed constant near the boundary, are extended by (the corresponding) constant on the complement of $\Omega$.

For $\delta \in \mathbb{R}$, the weighted norm $\|f\|_{L_{\delta}^{2}}^{2}:=\int_{\mathbb{R}^{n}}|f(x)|^{2}\left(1+|x|^{2}\right)^{\delta} d x$ is used. For $\mathbf{k}, \eta, \mathbf{l} \in \mathbb{R}^{n}$ with $\mathbf{k} \cdot \eta=\mathbf{k} \cdot \mathbf{l}=\mathbf{k} \cdot \eta=0$, and $|\eta|^{2}=\frac{|\mathbf{k}|^{2}}{4}+|\mathbf{l}|^{2}$, consider the vectors

$$
\begin{aligned}
& \xi_{1}(\eta, \mathbf{k}, \mathbf{l}):=\eta-i\left(\frac{\mathbf{k}}{2}+\mathbf{l}\right), \\
& \xi_{2}(\eta, \mathbf{k}, \mathbf{l}):=-\eta-i\left(\frac{\mathbf{k}}{2}-\mathbf{l}\right) .
\end{aligned}
$$

Note that $\xi_{1} \cdot \xi_{1}=\xi_{2} \cdot \xi_{2}=0,\left|\xi_{1}\right|^{2}=\left|\xi_{2}\right|^{2}=2\left(\left|\frac{\mathbf{k}}{2}\right|^{2}+|\mathbf{l}|^{2}\right)$, and $\xi_{1}+\xi_{2}=-i \mathbf{k}$. We restate their result in the variant below.

TheOREM 2.1 (Theorem 2.3 in [18]). Let $n \geq 3$ and $\sigma \in C^{1,1}(\bar{\Omega})$ be constant near the boundary. For $-1<\delta<0$ there are two constants $R, C>0$ dependent only on $\delta,\|\Delta \sqrt{\sigma} / \sqrt{\sigma}\|_{L^{\infty}(\Omega)}$, and $\Omega$ such that, for $\xi_{j} \in \mathbb{C}^{n}, j=1,2$ as in (2.1) with

$$
|\mathbf{l}|>R
$$


there exist $w\left(\cdot, \xi_{j}\right) \in H_{\text {loc }}^{1}\left(\mathbb{R}^{n}\right)$ solutions of $\nabla \cdot \sigma \nabla w\left(\cdot, \xi_{j}\right)=0$ in $\mathbb{R}^{n}$, of the form

$$
w\left(x, \xi_{j}\right)=e^{x \cdot \xi_{j}} \sigma^{-1 / 2}\left(1+\psi\left(x, \xi_{j}\right)\right)
$$

with

$$
\left\|\psi\left(\cdot, \xi_{j}\right)\right\|_{L_{\delta}^{2}} \leq \frac{C}{\left|\xi_{j}\right|}
$$

The main result which will be proven in section 4 is the following.

TheOREM 2.2. Let $\Omega \subset \mathbb{R}^{n}, n \geq 3$ be an open domain with $C^{1,1}$-boundary, $\sigma \in C^{1,1}(\bar{\Omega})$ be constant near the boundary, and $\epsilon \in C_{0}^{1,1}(\Omega)$. Recall the frequency differential map $\mathcal{D}$ defined in (1.4). For each $\mathbf{k} \in \mathbb{R}^{n}$, let $f_{j}:=\left.w\left(\cdot, \xi_{j}\right)\right|_{\partial \Omega}, j=1,2$ be the traces of the complex geometrical optics solutions in (2.2). Then

$$
\mathcal{F}\left[\frac{\nabla \cdot(\nabla \epsilon-\epsilon \nabla \ln \sigma)}{\sigma}\right](\mathbf{k})=\lim _{|\mathbf{l}| \rightarrow \infty}-2 i \int_{\partial \Omega} \mathcal{D}\left(f_{1}\right) f_{2} d s,
$$

where $\mathcal{F}$ denotes the Fourier transform.

We stress here that only the action of $\mathcal{D}$ on real valued functions is needed in Theorem 2.2 above.

If the Dirichlet-to-Neumann map $\Lambda_{\sigma}$ (at frequency $\omega=0$ ) is also known, then $\sigma$ can be recovered inside as shown in [14]. As a corollary to Theorem 2.2 one is also able to reconstruct $\epsilon$ inside.

Corollary 2.1. Let $\Omega \subset \mathbb{R}^{n}, n \geq 3$ be an open domain with smooth $C^{1,1}$ boundary. Assume $\sigma \in C^{1,1}(\bar{\Omega})$ constant near the boundary and $\epsilon \in C_{0}^{1,1}(\Omega)$. Then $\sigma$ and $\epsilon$ inside $\Omega$ can be reconstructed from knowledge of $\Lambda_{\sigma}$ and $\mathcal{D}$.

3. Analytic dependence in frequency. In this section we prove the analytic dependence in the frequency of $\omega \mapsto \Lambda_{\sigma+i \omega \epsilon}$ in the strong operator topology from $H^{1 / 2}(\Omega) \rightarrow H^{-1 / 2}(\Omega)$. The assumptions on the coefficients are slightly relaxed. By $C^{0,1}(\Omega)$ we denote the space of Lipschitz continuous maps.

TheOrEm 3.1. Let $\sigma \in L^{\infty}(\Omega)$ bounded away from zero and $\epsilon \in C^{0,1}(\Omega)$. Assume that $\omega$ lies in the frequency range (1.6). Then the Dirichlet problem (1.1) has a unique solution $u_{\omega} \in H^{1}(\Omega)$ with the following series representation:

$$
\begin{aligned}
& v_{\omega}(x):=\Re\left(u_{\omega}(x)\right)=\sum_{k=0}^{\infty} v_{2 k}(x) \omega^{2 k} \quad \text { and } \\
& h_{\omega}(x):=\Im\left(u_{\omega}(x)\right)=\sum_{k=1}^{\infty} h_{2 k-1}(x) \omega^{2 k-1},
\end{aligned}
$$

where the summation is convergent in the $H^{1}(\Omega), v_{0}$ is the solution to (1.3), and for $k=1,2, \ldots$ the following recurrence holds:

$$
\left\{\begin{array}{l}
\nabla \cdot\left(\sigma \nabla h_{2 k-1}\right)=-\nabla \cdot\left(\epsilon \nabla v_{2(k-1)}\right) \quad \text { in } \Omega, \\
\nabla \cdot\left(\sigma \nabla v_{2 k}\right)=\nabla \cdot\left(\epsilon \nabla h_{2 k-1}\right) \quad \text { in } \Omega, \\
\left.h_{2 k-1}\right|_{\partial \Omega}=\left.v_{2 k}\right|_{\partial \Omega}=0 .
\end{array}\right.
$$

Assuming the recurrences in (3.2) hold we establish first some basic estimates.

Copyright $@$ by SIAM. Unauthorized reproduction of this article is prohibited. 
Lemma 3.1. Let $v_{2 k}$ and $h_{2 k-1}, k=1,2, \ldots$ be defined in (3.2). Then, for $k=1,2, \ldots$, we have

$$
\left[\int_{\Omega} \sigma\left|\nabla v_{2 k}\right|^{2} d x\right]^{\frac{1}{2}} \leq\left\|\frac{\epsilon}{\sigma}\right\|_{L^{\infty}(\Omega)}^{2 k}\left[\int_{\Omega} \sigma\left|\nabla v_{0}\right|^{2} d x\right]^{\frac{1}{2}}
$$

and

$$
\left[\int_{\Omega} \sigma\left|\nabla h_{2 k-1}\right|^{2} d x\right]^{\frac{1}{2}} \leq\left\|\frac{\epsilon}{\sigma}\right\|_{L^{\infty}(\Omega)}^{2 k-1}\left[\int_{\Omega} \sigma\left|\nabla v_{0}\right|^{2} d x\right]^{\frac{1}{2}}
$$

Proof. Let us fix a positive natural number $k$. From (3.2) the divergence theorem implies that

$$
\int_{\Omega} \sigma\left|\nabla h_{2 k-1}\right|^{2} d x=-\int_{\Omega} \epsilon \nabla v_{2 k-2} \cdot \nabla h_{2 k-1} d x .
$$

Cauchy's inequality applied to the right-hand side above yields

$$
\int_{\Omega} \sigma\left|\nabla h_{2 k-1}\right|^{2} d x \leq\left\|\frac{\epsilon}{\sigma}\right\|_{L^{\infty}(\Omega)}\left[\int_{\Omega} \sigma\left|\nabla v_{2 k-2}\right|^{2} d x\right]^{\frac{1}{2}}\left[\int_{\Omega} \sigma\left|\nabla h_{2 k-1}\right|^{2} d x\right]^{\frac{1}{2}},
$$

and thus

$$
\left[\int_{\Omega} \sigma\left|\nabla h_{2 k-1}\right|^{2} d x\right]^{\frac{1}{2}} \leq\left\|\frac{\epsilon}{\sigma}\right\|_{L^{\infty}(\Omega)}\left[\int_{\Omega} \sigma\left|\nabla v_{2 k-2}\right|^{2} d x\right]^{\frac{1}{2}} .
$$

Similarly we obtain

$$
\left[\int_{\Omega} \sigma\left|\nabla v_{2 k}\right|^{2} d x\right]^{\frac{1}{2}} \leq\left\|\frac{\epsilon}{\sigma}\right\|_{L^{\infty}(\Omega)}\left[\int_{\Omega} \sigma\left|\nabla h_{2 k-1}\right|^{2} d x\right]^{\frac{1}{2}} .
$$

By induction, the estimates (3.3) and (3.4) follow.

Proof of Theorem 3.1. The coupled system (1.5) is equivalent to the two elliptic equations

$$
\nabla \cdot\left(\sigma \nabla v_{\omega}\right)=\nabla \cdot\left(\omega \epsilon \nabla h_{\omega}\right) \quad \text { in } \Omega
$$

and

$$
\nabla \cdot\left(\sigma \nabla h_{\omega}\right)=-\nabla \cdot\left(\omega \epsilon \nabla v_{\omega}\right) \quad \text { in } \Omega .
$$

We seek solutions in the ansatz

$$
v(x, \omega):=\sum_{k=0}^{\infty} v_{k}(x) \omega^{k} \quad \text { and } \quad h(x, \omega):=\sum_{k=0}^{\infty} h_{k}(x) \omega^{k} .
$$

Let us assume first that the series representations in (3.9) are convergent in $H^{1}(\Omega)$. If (3.7) and (3.8) are satisfied, then

$$
0=\nabla \cdot\left(\sigma \nabla v_{0}\right)+\sum_{k=0}^{\infty} \nabla \cdot\left(\sigma \nabla v_{k+1}-\epsilon \nabla h_{k}\right) \omega^{k+1}
$$

Copyright $@$ by SIAM. Unauthorized reproduction of this article is prohibited. 
and

$$
0=\nabla \cdot\left(\sigma \nabla h_{0}\right)+\sum_{k=0}^{\infty} \nabla \cdot\left(\sigma \nabla h_{k+1}+\epsilon \nabla v_{k}\right) \omega^{k+1},
$$

where the divergence is taken in the weak sense. In particular we obtain

$$
\nabla \cdot\left(\sigma \nabla v_{0}\right)=0, \quad \nabla \cdot\left(\sigma \nabla h_{0}\right)=0 \quad \text { in } \Omega,
$$

and, for $k=0,1,2, \ldots$,

$$
\nabla \cdot\left(\sigma \nabla v_{k+1}\right)=\nabla \cdot\left(\epsilon \nabla h_{k}\right), \quad \nabla \cdot\left(\sigma \nabla h_{k+1}\right)=-\nabla \cdot\left(\epsilon \nabla v_{k}\right) \quad \text { in } \Omega .
$$

By our assumption, both series are convergent in $H^{1}(\Omega)$, and their sums have well-defined traces in $H^{1 / 2}(\partial \Omega)$, which are the corresponding sums of the traces of the terms. Now $v(x, \omega)=f(x)$ and $h(x, \omega)=0$ for $x \in \partial \Omega$ yield $\left.v_{0}\right|_{\partial \Omega}=f$ and $\left.h_{0}\right|_{\partial \Omega}=0$, and, for $k=1,2, \ldots$,

$$
\left.h_{2 k-1}\right|_{\partial \Omega}=\left.v_{2 k}\right|_{\partial \Omega}=0 \quad \text { on } \partial \Omega .
$$

Note that $v_{0}$ is the solution of $(1.3)$ and $h_{0} \equiv 0$.

Conversely, for $f \in H^{1 / 2}(\partial \Omega)$, let $v_{0}$ be the solution of (1.3) and define two sequences of functions $\left\{v_{k}\right\}_{0}^{\infty}$ and $\left\{h_{k}\right\}_{1}^{\infty}$ via the recurrence (3.2). From Lemma 3.1 it follows that for any $k=1,2, \ldots$,

$$
\begin{aligned}
\left\|\nabla v_{2 k}\right\|_{L^{2}(\Omega)} \omega^{2 k} & \leq \frac{1}{\sqrt{m}}\left[\int_{\Omega} \sigma\left|\nabla v_{2 k}\right|^{2} d x\right]^{\frac{1}{2}} \omega^{2 k} \\
& \leq \frac{1}{\sqrt{m}}\left\|\frac{\omega \epsilon}{\sigma}\right\|_{L^{\infty}(\Omega)}^{2 k}\left[\int_{\Omega} \sigma\left|\nabla v_{0}\right|^{2} d x\right]^{\frac{1}{2}} \\
& \leq \sqrt{\frac{M}{m}}\left\|\nabla v_{0}\right\|_{L^{2}(\Omega)}\left\|\frac{\omega \epsilon}{\sigma}\right\|_{L^{\infty}(\Omega)}^{2 k}
\end{aligned}
$$

and

$$
\begin{aligned}
\left\|\nabla h_{2 k-1}\right\|_{L^{2}(\Omega)} \omega^{2 k-1} & \leq \frac{1}{\sqrt{m}}\left[\int_{\Omega} \sigma\left|\nabla h_{2 k-1}\right|^{2} d x\right]^{\frac{1}{2}} \omega^{2 k-1} \\
& \leq \frac{1}{\sqrt{m}}\left\|\frac{\omega \epsilon}{\sigma}\right\|_{L^{\infty}(\Omega)}^{2 k-1}\left[\int_{\Omega} \sigma\left|\nabla v_{0}\right|^{2} d x\right]^{\frac{1}{2}} \\
& \leq \sqrt{\frac{M}{m}}\left\|\nabla v_{0}\right\|_{L^{2}(\Omega)}\left\|\frac{\omega \epsilon}{\sigma}\right\|_{L^{\infty}(\Omega)}^{2 k-1} .
\end{aligned}
$$

If $\omega$ satisfies (1.6), the $H^{1}(\Omega)$-convergence of the series well defines $v(x, \omega)$ and $h(x, \omega)$ in $(3.9)$ to be the unique solutions of $(1.5)$.

The result below further clarifies the frequency differential operator $\mathcal{D}$ in (1.4).

Corollary 3.2. Let $\epsilon \in C^{0,1}(\bar{\Omega})$, and $\sigma \in L^{\infty}(\Omega)$ with essinf $\Omega_{\Omega} \sigma>0$. For $f, g \in H^{1 / 2}(\partial \Omega)$ real valued we have

$$
\mathcal{D}(f+i g)=i \epsilon \frac{\partial v_{0}}{\partial \mathbf{n}}+i \sigma \frac{\partial h_{1}}{\partial \mathbf{n}}
$$

Copyright $@$ ( ) by SIAM. Unauthorized reproduction of this article is prohibited. 
where $v_{0}$ solves $\nabla \cdot\left(\sigma \nabla v_{0}\right)=0$ in $\Omega$, and $\left.v_{0}\right|_{\partial \Omega}=f+i g$, and $h_{1}$ solves

$$
\nabla \cdot\left(\sigma \nabla h_{1}\right)=-\nabla \cdot \epsilon \nabla v_{0} \text { in } \Omega \text { and }\left.h_{1}\right|_{\partial \Omega}=0 .
$$

Proof. If $g=0$ the corollary follows directly from the definition (1.4) and the fact that $\left.\frac{d}{d \omega} \frac{\partial h_{\omega}}{\partial \mathbf{n}}\right|_{\omega=0}=\frac{\partial h_{1}}{\partial \mathbf{n}}$, with $h_{1}$ defined in the recurrence (3.2). If $g$ is arbitrary, the result follows from the complex linearity of the two terms in the right-hand side of (3.12).

From Theorem 3.1 it follows that $v_{0}$ is the zeroth order term of the series expansion of the real part $v_{\omega}$ and $h_{1}$ is the first order term of the series expansion of the imaginary part $h_{\omega}$. Moreover, when $\omega \ll 1$ is small, and $\omega_{1}, \omega_{2}=O(\omega)$, we have

$$
\frac{v_{\omega_{1}}-v_{\omega_{2}}}{\omega_{1}-\omega_{2}}=v_{2}\left(\omega_{1}+\omega_{2}\right)+O\left(\omega^{3}\right) \text { and } \frac{h_{\omega_{1}}-h_{\omega_{2}}}{\omega_{1}-\omega_{2}}=h_{1}+O\left(\omega^{2}\right),
$$

where $h_{1}$ and $v_{2}$ satisfy the Poisson's problems

$$
\left\{\begin{array}{l}
\nabla \cdot\left(\sigma \nabla h_{1}\right)=-\nabla \cdot\left(\epsilon \nabla v_{0}\right) \quad \text { in } \Omega, \\
\nabla \cdot\left(\sigma \nabla v_{2}\right)=\nabla \cdot\left(\epsilon \nabla h_{1}\right) \quad \text { in } \Omega, \\
\left.h_{1}\right|_{\partial \Omega}=\left.v_{2}\right|_{\partial \Omega}=0 .
\end{array}\right.
$$

4. Proof of Theorem 2.2 and its corollary. For $j=1,2$ let $w_{j}(x):=w\left(x ; \xi_{j}\right)$ be the complex geometrical optics (CGO) solutions corresponding to a fix vector $\mathbf{k} \in \mathbb{R}^{n}$ as provided by Theorem 2.1. For $w_{1}$ in the CGO above, let $h_{1}$ be the solution of the Poisson equation

$$
\nabla \cdot\left(\sigma \nabla h_{1}\right)=-\nabla \cdot\left(\epsilon \nabla w_{1}\right) \quad \text { in } \Omega \text { and }\left.h_{1}\right|_{\partial \Omega}=0 .
$$

First we carry out the calculation without the assumption that $\epsilon=0$ near the boundary to emphasize the fact that knowledge of $\epsilon$ and its normal derivative at the boundary suffices.

Following the explicit formula for $\mathcal{D}$ in Corollary 3.2 we obtain

$$
\begin{aligned}
\int_{\partial \Omega} \mathcal{D}\left(w_{1}\right) w_{2} d s & =i \int_{\partial \Omega}\left(\epsilon \frac{\partial w_{1}}{\partial \mathbf{n}}+\sigma \frac{\partial h_{1}}{\partial \mathbf{n}}\right) w_{2} d s \\
& =i \int_{\Omega} \sigma \nabla h_{1} \cdot \nabla w_{2}+\epsilon \nabla w_{1} \cdot \nabla w_{2} d x
\end{aligned}
$$

Since $h_{1}=0$ on $\partial \Omega$ and $w_{2}$ solves the conductivity equation $\nabla \cdot \sigma \nabla w_{2}=0$, the first integral on the right-hand side of (4.1) is zero so that

$$
\int_{\partial \Omega} \mathcal{D}\left(w_{1}\right) w_{2} d s=i \int_{\Omega} \epsilon \nabla w_{1} \cdot \nabla w_{2} d x
$$

Now use $2 \nabla w_{1} \cdot \nabla w_{2}=\left[\Delta\left(w_{1} w_{2}\right)-\left(w_{1} \Delta w_{2}+w_{2} \Delta w_{1}\right)\right]$ and the fact that the $w_{j}$ 's also solve

$$
\Delta w_{j}+\nabla \ln \sigma \cdot \nabla w_{j}=0 \quad \text { in } \Omega,
$$

to obtain

$$
\begin{aligned}
\int_{\Omega} \epsilon \nabla w_{1} \cdot \nabla w_{2} d x= & \frac{1}{2} \int_{\partial \Omega}\left(\epsilon \frac{\partial\left(w_{1} w_{2}\right)}{\partial \mathbf{n}}-\frac{\partial \epsilon}{\partial \mathbf{n}}\left(w_{1} w_{2}\right)\right) d s \\
& +\frac{1}{2} \int_{\Omega}\left[(\Delta \epsilon)\left(w_{1} w_{2}\right)+\epsilon \nabla \ln \sigma \cdot \nabla\left(w_{1} w_{2}\right)\right] d x
\end{aligned}
$$

Copyright $@$ by SIAM. Unauthorized reproduction of this article is prohibited. 
Using the Green's formula in the last integral and the assumption that $\sigma$ is constant near the boundary we obtain

$$
\begin{aligned}
\int_{\Omega} \epsilon \nabla w_{1} \cdot \nabla w_{2} d x= & \frac{1}{2} \int_{\partial \Omega}\left(\epsilon \frac{\partial\left(w_{1} w_{2}\right)}{\partial \mathbf{n}}-\frac{\partial \epsilon}{\partial \mathbf{n}}\left(w_{1} w_{2}\right)\right) d s \\
& +\frac{1}{2} \int_{\Omega} \nabla \cdot(\nabla \epsilon-\epsilon \nabla \ln \sigma)\left(w_{1} w_{2}\right) d x .
\end{aligned}
$$

From (4.2) and (4.3) we have that

$$
\begin{aligned}
\int_{\Omega} \nabla \cdot(\nabla \epsilon-\epsilon \nabla \ln \sigma) w_{1} w_{2} d x= & -2 i \int_{\partial \Omega} \mathcal{D}\left(w_{1}\right) w_{2} d s \\
& -\int_{\partial \Omega}\left(\epsilon \frac{\partial\left(w_{1} w_{2}\right)}{\partial \mathbf{n}}-\frac{\partial \epsilon}{\partial \mathbf{n}}\left(w_{1} w_{2}\right)\right) d s .
\end{aligned}
$$

If we now use the assumption of $\epsilon$ being support in $\Omega$, (4.4) further simplifies to

$$
\int_{\Omega} \nabla \cdot(\nabla \epsilon-\epsilon \nabla \ln \sigma) w_{1} w_{2} d x=-2 i \int_{\partial \Omega} \mathcal{D}\left(w_{1}\right) w_{2} d s .
$$

By the choice of $\xi_{j}$ 's in the complex geometrical optics $w_{j}$ 's, we have

$$
\int_{\Omega} \frac{\nabla \cdot(\nabla \epsilon-\epsilon \nabla \ln \sigma)}{\sigma} \mathbf{e}^{-i x \cdot \mathbf{k}}\left(1+\psi\left(x, \xi_{1}\right)\right)\left(1+\psi\left(x, \xi_{2}\right)\right) d x=-2 i \int_{\partial \Omega} \mathcal{D}\left(w_{1}\right) w_{2} d s .
$$

Since the integrant in the left-hand side above is supported in $\Omega$, the integral can be taken over the entire space $\mathbb{R}^{n}$. The decay estimates (2.3) then yield

$$
\mathcal{F}\left[\frac{\nabla \cdot(\nabla \epsilon-\epsilon \nabla \ln \sigma)}{\sigma}\right](\mathbf{k})=\lim _{|1| \rightarrow \infty}-2 i \int_{\partial \Omega} \mathcal{D}\left(w_{1}\right) w_{2} d s
$$

where $\mathcal{F}$ is the Fourier transform in $\mathbb{R}^{n}$. This completes our proof of Theorem 2.2.

The proof of Corollary 2.1 relies on the results in [14] which show that the traces of the geometrical optics solutions used in Theorem (2.2) can been recovered from a singular integral equation at the boundary: Since $\sigma \in C^{1,1}(\bar{\Omega})$ is recovered inside from $\Lambda_{\sigma}$, we may assume without loss of generality that $\sigma=1$ near the boundary. Then Lemmas 2.7 and $2.12(\mathrm{~b})$ in [14] yield that the traces $f_{j}:=\left.w\left(\cdot, \xi_{j}\right)\right|_{\partial \Omega}$ at the boundary are the unique solutions to the equation

$$
f_{j}=e^{i x \cdot \xi_{j}}-\left(S_{\xi_{j}} \Lambda_{\gamma}-B_{\xi_{j}}-\frac{1}{2} I\right) f_{j}, \quad j=1,2,
$$

where the boundary operators $S_{\xi}$ and $B_{\xi}$ are the single and double layer potentials

$$
S_{\xi} f(x)=\int_{\partial \Omega} G_{\xi}(x, y) f(y) d s \text { and } B_{\xi} f(x)=p \cdot v \cdot \int_{\partial \Omega} \frac{\partial G_{\xi}}{\partial \mathbf{n}}(x, y) f(y) d s
$$

associated with the Fadeev-Green kernel

$$
G_{\xi}(x)=\frac{e^{i x \cdot \xi}}{(2 \pi)^{n}} \int_{\mathbb{R}^{n}} \frac{e^{i x \cdot \eta}}{\eta^{2}+2 \xi \cdot \eta} d \eta .
$$

Once the traces of $w_{1}$, and $w_{2}$ are determined, the right-hand side of (2.4) is determined. By Fourier inversion, we then determine the essentially bounded function

$$
Q[\sigma, \epsilon]:=\frac{\nabla \cdot(\nabla \epsilon-\epsilon \nabla \ln \sigma)}{\sigma} .
$$

Copyright (c) by SIAM. Unauthorized reproduction of this article is prohibited. 
With $\sigma$ and $Q$ known, the permittivity $\epsilon$ is the unique solution of the Dirichlet problem

$$
\Delta \epsilon-\nabla \epsilon \cdot \nabla \ln (\sigma)-\epsilon \Delta \ln (\sigma)=Q \sigma \text { in } \Omega,\left.\quad \epsilon\right|_{\partial \Omega}=0 .
$$

This finishes the proof of Corollary 2.1.

5. Concluding remarks. We formulated a Calderón-type problem using frequency differential $\mathcal{D}:=\left.\frac{d \Lambda_{\gamma}}{d \omega}\right|_{\omega=0}$ of the Dirichlet-to-Neumann map at $\omega=0$. Provided that $\sigma$ is (an unknown) constant near the boundary and $\epsilon$ is supported in $\Omega$, we showed that the frequency differential uniquely determines $Q$ in (4.7) relating the conductivity $\sigma$ to the permittivity $\epsilon$. However, if the Dirichlet-to-Neumann map at $\omega=0$ is also available, then $\sigma$ and $\epsilon$ can be recovered inside. We note here that $\epsilon$ need not be supported in $\Omega$, since the quantity $Q$ in (4.7) can still be recovered if $\left.\epsilon\right|_{\partial \Omega}$ and its normal derivative $\frac{\partial \epsilon}{\partial \mathbf{n}}$ are known at the boundary, according to (4.4).

Our results yield the following effect of the admittivity $\sigma+i \omega \epsilon$ on the complex voltage potential $u_{\omega}$. The real part $\Re\left(u_{\omega}\right)$ is influenced mainly by the conductivity $\sigma$, whereas the imaginary part $\Im\left(u_{\omega}\right)$ is influenced by the combination $Q[\sigma, \epsilon]$ in (4.7).

There are infinitely many pairs $\sigma, \epsilon$ which yield the same quantity $Q$ in (4.7). More precisely, let $Q \in L^{\infty}(\Omega)$ be in the range of the combination in (4.7). For an arbitrary $f \in C^{1,1}(\bar{\Omega})$, let $\sigma_{f}$ be any solution of the transport equation

$$
\nabla \ln \sigma_{f} \cdot \nabla f=Q-\Delta f .
$$

Then the pair $\left(\sigma_{f}, \epsilon\right)$ with $\epsilon=f \sigma_{f}$ yields the same $Q$, independent of $f$.

If the actual value of $\sigma$ at the boundary is not known, since $Q[\lambda \sigma, \lambda \epsilon]=Q[\sigma, \epsilon]$ for any $\lambda>0$, the recovered quantity is not sensitive to the contrast in the pair of coefficients. However, the boundary data $\mathcal{D}$ can distinguish the difference in scale between the conductivity $\sigma$ and the permittivity $\epsilon$ since $Q[\sigma, \lambda \epsilon]=\lambda Q[\sigma, \epsilon]$.

In practice the angular frequency $\omega$ is not arbitrarily small. However, due the scaling $\gamma=\sigma+i(t \omega) \frac{\epsilon}{t}$, we apply the results above to $t \omega$, with $t$ small. This scaling is meaningful at angular frequencies of up to a few $\mathrm{kHz}$, where the scaling factor is the permittivity of the vacuum $\epsilon_{0}=8.8 \times 10^{-12} \mathrm{~F} / \mathrm{m}$, since then $\omega \epsilon_{0}$ is still numerically small.

From a numerical perspective, (3.13) shows a difference in scale (of order 1) between the real and imaginary parts of the complex voltage potential at small frequency (or $\epsilon_{0} \omega$ as explained above). They imply that $\mathcal{D}$ is approximated at $O\left(\omega^{2}\right)$ by the difference quotient at two small frequencies without a need to distinguish the real from the imaginary part of the voltage potential.

Following from (1.7), in fdEIT it is the quotient $\left.\frac{\epsilon}{\sigma^{2}}\right|_{\partial \Omega}$ at the boundary which scales the boundary information about the admitivitty inside. In particular, when $\left.\epsilon\right|_{\partial \Omega}=0$ it is only the imaginary part of the voltage potential which carries the information about the coefficients from inside to the frequency differential data at the boundary. In such a case we can still expect to recover the quantity $Q$ in (4.7).

While the formulated problem is still severely ill-posed, these theoretical results are expected to help in understanding the quantitative feature of fdEIT.

Acknowledgments. This work was done during the first author's sabbatical visit to A. Tamasan at the University of Central Florida. The first author would like to thank them both for the hospitality. 


\section{REFERENCES}

[1] K. Astala and L. PÄIvÄRInta, Calderón's inverse conductivity problem in the plane, Ann. of Math. (2), 163 (2006), pp. 265-299.

[2] R.H. BAYFord, Bioimpedance tomography (electrical impedance tomography), Annu. Rev. Biomed. Eng., 8 (2006), pp. 63-91.

[3] L. Borcea, EIT Electrical impedance tomography, Inverse Problems, 18 (2002), pp. R99-R136.

[4] A.L. Bukhgeim, Recovering a potential from Cauchy data in the two-dimensional case, J. Inverse Ill-Posed Problems, 16 (2008), pp. 19-33.

[5] A.P. Calderón, On an inverse boundary value problem, in Seminar on Numerical Analysis and its Applications to Continuum Physics, Soc. Brasiliera de Matematica, Rio de Janeiro, 1980, pp. 65-73.

[6] M. Cheney, D. Isaacson, and J C. Newell, Electrical impedance tomography, SiAM Rev., 41 (1999), pp. 85-101.

[7] E. Francini, Recovering a complex coefficient in a planar domain from the Dirichlet-toNeumann map, Inverse Problems, 16 (2000), p. 107.

[8] D. Holder, Electrical Impedance Tomography: Methods, History and Applications, IOP, Bristol, UK, 2005.

[9] S. Kim, J. Lee, J.K. Seo, E. J. Woo, And H. ZRIBI, Multifrequency trans-admittance scanner: Mathematical framework and feasibility, SIAM J. Appl. Math., 69 (2008), pp. 22-36.

[10] S. KIm, J.K. SEO, AND T. HA, A nondestructive evaluation method for concrete voids: Frequency differential electrical impedance scanning, SIAM J. Appl. Math., 69 (2009), pp. 1759-1771.

[11] S. KIM, Assessment of breast tumor size in electrical impedance scanning, Inverse Problems, 28 (2012), 025004.

[12] S. Kim, E.J. Lee, E.J. Woo, And J.K. SeO, Asymptotic analysis of the membrane structure to sensitivity of frequency-difference electrical impedance tomography, Inverse Problems, 28 (2012), 075004.

[13] R.V. Kohn and M. Vogelius, Determining conductivity by boundary measurements, Comm. Pure Appl. Math., 37 (1984), pp. 113-123.

[14] A. Nachman, Reconstructions from boundary measurements, Ann. of Math. (2), 128 (1988), pp. 531-576.

[15] A. NACHMAn, Global uniqueness for a two-dimensional inverse boundary value problem, Ann. of Math. (2), 143 (1996), pp. 71-96.

[16] J.K. Seo, J. LeE, S.W. Kim, H. ZRIBI, AND E.J. Woo, Frequency-difference electrical impedance tomography (fdEIT): Algorithm development and feasibility study, Inverse Problems, 29 (2008), pp. 929-944.

[17] J.K. Seo, B. Harrach, And E.J. Woo, Recent progress on frequency difference electrical impedance tomography, ESAIM Proc., 26 (2009), pp. 150-161.

[18] J. Sylvester and G. Uhlmann, A global uniqueness theorem for an inverse boundary value problem, Ann. of Math. (2), 125 (1987), pp. 153-169.

[19] G. Uhlmann, Electrical impedance tomography and Calderón's problem, Inverse Problems, 25 (2009), pp. 1-39.

Copyright (c) by SIAM. Unauthorized reproduction of this article is prohibited. 